\title{
Optic Nerve Lesion Length at the Acute Phase of Optic Neuritis Is Predictive of Retinal Neuronal Loss
}

\author{
Mickael Denis, MD, Jean-Philippe Woillez, MD, PhD, Vasily M. Smirnov, MD, Elodie Drumez, Julien Lannoy, MD, \\ Julie Boucher, MD, Mickael Zedet, MD, Jean-Pierre Pruvo, MD, PhD, Julien Labreuche, Helene Zephir, MD, PhD, \\ Xavier Leclerc, MD, PhD, and Olivier Outteryck, MD, PhD
}

Neurol Neuroimmunol Neuroinflamm 2022;9:e1135. doi:10.1212/NXI.0000000000001135

\section{Abstract \\ Background and Objectives}

Acute optic neuritis (ON) is a classical presenting symptom of multiple sclerosis (MS), neuromyelitis optica spectrum disorders (NMOSD), and anti-MOG-associated disorders. The resulting visual impairment is variable and can be severe. Clinicians are in need of predictive biomarkers to optimize the management of acute ON. In this longitudinal study (IRMANO, NCT03651662), we evaluated the ability of optic nerve lesion length measured on MRI at the acute phase of $\mathrm{ON}$ to predict retinal neuro-axonal loss and visual impairment at a chronic stage.

\section{Methods}

We conducted a longitudinal study (IRMANO, NCT03651662) of patients who presented a clinical episode of ON ( $\leq 8$ weeks). All patients underwent a retinal optical coherence tomography (OCT) and a brain/optic nerve MRI, including 3D double-inversion recovery (DIR) sequence at the acute phase of $\mathrm{ON}$ and 12 months later. Primary outcomes were optic nerve DIR hypersignal lesion length, macular ganglion cell-inner plexiform layer (GCIPL) volume measured on OCT, and low-contrast monocular visual acuity (LCMVA).

\section{Results}

The study group included 51 patients ( 33 women, mean age of 32.4 years \pm 7.9 ). We recruited patients with a clinically isolated syndrome $(\mathrm{n}=20)$, a relapsing-remitting MS $(\mathrm{n}=23)$, an isolated ON $(n=6)$, and a first clinical episode of $\operatorname{NMOSD}(n=2)$. Optic nerve DIR hypersignal was observed in all but 1 symptomatic optic nerves. At inclusion, the mean optic nerve lesion length (in $\mathrm{mm}$ ) was $12.35 \pm 5.98$. The mean GCIPL volume (in $\mathrm{mm}^{3}$ ) significantly decreased between inclusion $(1.90 \pm 0.18)$ and $\mathrm{M}_{12}(1.67 \pm 0.21 ; p<0.0001)$. Optic nerve lesion length at inclusion was significantly associated with GCIPL thinning (estimate \pm SD; $-0.012 \pm 0.004 ; p=0.0016)$ and LCMVA at $\mathrm{M}_{12}(0.016 \pm 0.003 ; p<0.001)$. Optic nerve lesion length significantly increased at $\mathrm{M}_{12}(15.76 \pm 8.70 ; p=0.0007)$. The increase in optic nerve lesion length was significantly associated with the GCIPL thinning between inclusion and $\mathrm{M}_{12}$ $(-0.012 \pm 0.003 ; p=0.0011)$.

\section{Discussion}

At the acute phase of $\mathrm{ON}$, optic nerve lesion length is an imaging biomarker predictive of retinal neuro-axonal loss and chronic visual impairment, which can help to stratify future therapeutic strategies in acute $\mathrm{ON}$.

\author{
Correspondence \\ Dr. Outteryck \\ olivier.outteryck@chru-lille.fr
}

\section{MORE ONLINE}

(II) Class of Evidence

Criteria for rating

therapeutic and diagnostic

studies

NPub.org/coe 


\section{Glossary}

3D-DIR = 3D double-inversion recovery; Abs = aquaporin 4 antibodies; ANCOVA $=$ analysis of covariance; CIS = clinically isolated syndrome; DMT = disease-modifying therapy; FU = follow-up; GCIPL = ganglion-cells-inner plexiform layer; IETD = intereye retinal thickness difference; INL = inner nuclear layer; LCMVA = low-contrast monocular visual acuity; MME = microcystic macular edema; MOGAD = MOG-associated disorder; $\mathbf{M S}=$ multiple sclerosis; NMOSD = neuromyelitis optica spectrum disorder; $\mathbf{O C T}=$ optical coherence tomography; $\mathbf{O N}=$ optic neuritis; $\mathbf{p R N F L}=$ peripapillary retinal nerve fiber layer; RRMS = relapsing-remitting multiple sclerosis; VF = visual field.

\section{Classification of Evidence}

This study provides Class I evidence that optic nerve lesion length measured on MRI during the acute phase of a first episode of $\mathrm{ON}$ is associated with long-term retinal neuro-axonal loss and visual impairment.

Acute optic neuritis $(\mathrm{ON})$ is a classical presenting symptom of multiple sclerosis (MS) where it accounts for $25 \%$ of first demyelinating events. Occurrence of $\mathrm{ON}$ during the disease is approximately $70 \% .{ }^{1} \mathrm{ON}$ is also one of the most frequent clinical manifestations of neuromyelitis optica spectrum disorders (NMOSD) and myelin oligodendrocyte glycoprotein-associated disorders (MOGAD). It could also be idiopathic. ${ }^{2}$

Some therapeutic strategies can be proposed in acute ON, including corticosteroids, plasmapheresis, and IV immunoglobulins. Most studies have not demonstrated that the timing of steroid administration makes a long-term visual difference after ON, but some studies suggested that timing of relapse treatment, as corticosteroids administration, may be important in NMOSD. ${ }^{3,4}$ In addition, some therapeutics that may promote remyelination are under evaluation. ${ }^{5}$ Clinicians are searching for predictive and reliable biomarkers to develop new therapeutic algorithms and to optimize the management of acute $\mathrm{ON}$.

Optic nerve MRI is challenging but remains a performing tool to detect optic nerve involvement in demyelinating diseases. To detect acute ON lesion with MRI, the best diagnostic strategy is by using $\mathrm{T} 2$-weighted sequences including both water and fat suppression. ${ }^{6}$ Among sequences including water and fat suppression, 3D double-inversion recovery (3D-DIR) sequence is one of the most sensitive., ${ }^{7,8}$ With such a sequence, we were able to detect many asymptomatic optic nerve lesions in clinically isolated syndrome ${ }^{9}$ (CIS) and MS. ${ }^{10,11}$ Diagnostic accuracy of 3D-DIR sequence for the detection of demyelinating optic nerve lesion(s) is very high, ${ }^{7}$ and intraobserver and interobserver agreement are very good for optic nerve DIR hypersignal length measurement. ${ }^{10}$ At a chronic stage, optic nerve DIR hypersignal has been associated with lower ganglion cell-inner plexiform layer (GCIPL) volumes and higher visual impairment. ${ }^{10,11}$ However, the predictive value of optic nerve DIR hypersignal length measured at the acute phase has not been evaluated yet.

Optic nerve lesion length or optic nerve gadolinium enhancement length has been previously suggested as a potential predictive biomarker of visual impairment, ${ }^{12-14}$ notably in NMO, ${ }^{3,15}$ but other studies did not confirm such results or did not show findings that may support this hypothesis. ${ }^{16-21}$

The aim of this study is to evaluate the association between the length of the optic nerve lesion measured on MRI during the acute phase of a first episode of $\mathrm{ON}$ and the long-term retinal neuro-axonal loss and visual impairment.

\section{Methods}

IRMANO study (Imagerie par Résonance Magnétique au stade Aigu de la Névrite Optique; NCT03651662) was a longitudinal and prospective cohort study conducted between February 2016 and February 2020, which enrolled 51 patients at Lille MS center (France).

\section{Participants}

Consenting adults (aged 18-65 years) diagnosed with a history of acute $\mathrm{ON}$ in the last 8 weeks were prospectively recruited. ON diagnosis was made on the basis of suggestive clinical symptoms (loss of vision and dyschromatopsia) and abnormal neuroophthalmologic examination, including visual-evoked potentials (P100 latency increasing) and visual fields (VFs; VF defect) if the demyelinating disease was not known. If the patient already experienced an already known demyelinating disease of the CNS, such as MS according to McDonald revised 2017 criteria, ${ }^{22}$ NMOSD according to 2015 International consensus criteria, ${ }^{23}$ or MOGAD, ${ }^{24} \mathrm{ON}$ diagnosis was made on the basis of typical clinical symptoms ${ }^{2}$ by a senior neurologist specialized in the management and treatment of CNS demyelinating diseases for more than 2 years and subsequently confirmed by a neuro-ophthalmologist. Patients with a unilateral history of $\mathrm{ON}$ may be included, but recent acute $\mathrm{ON}$ must concern the fellow eye. Included patients presented with a recent clinical episode of acute ON. Data on sex, age at $\mathrm{ON}$ onset, type of demyelinating disease, disease duration, and delay between first visual symptoms and inclusion in the study were collected. Some patients were already under diseasemodifying therapy (DMT) at the time of MRI/optical coherence tomography (OCT) evaluation. Some others started a DMT during the follow-up (FU). Data on DMT and particularly on fingolimod initiation during the study were collected because 

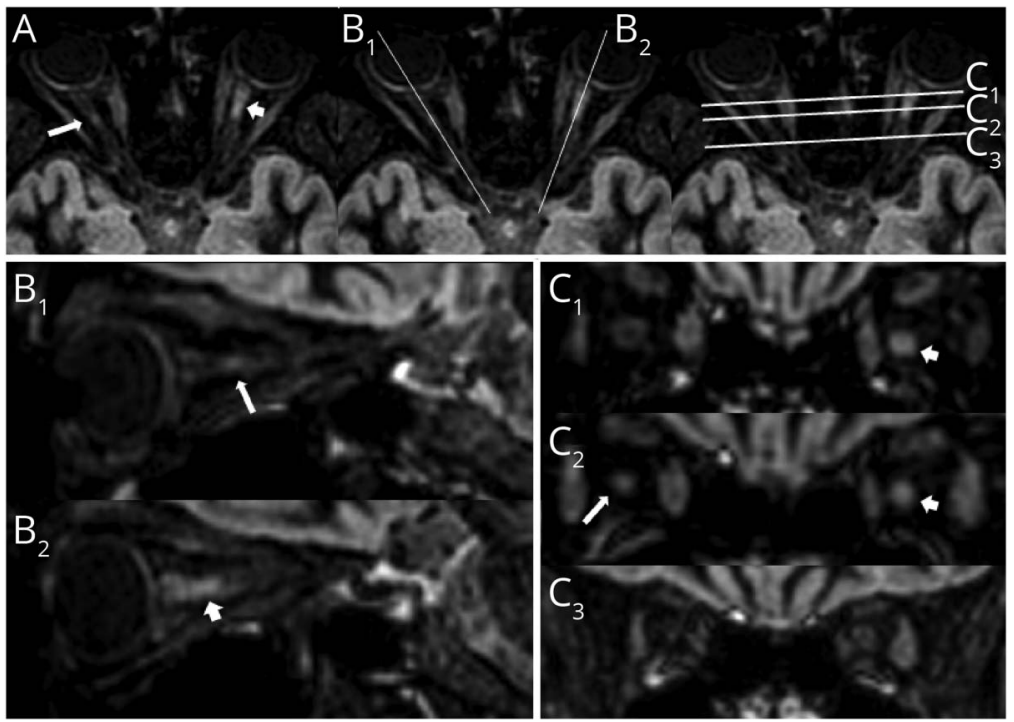

(A) Patient with CIS presenting a left clinical episode of acute optic neuritis. A symptomatic optic nerve lesion (arrowhead) and a right asymptomatic optic nerve lesion (white arrow) were detected on 3D-Double-Inversion Recovery MR sequence. MRI reconstruction of left and right optic nerves showing symptomatic (arrowheads) and asymptomatic (white arrows) lesions in sagittal optic nerve planes (B) and coronal planes (C). CIS = clinically isolated syndrome

fingolimod treatment can lead to increased macular volume in $\mathrm{MS}^{25}$ Subjects with other retinal pathology and/or severe ametropia ( $\geq 6$ diopters) were excluded. Patients with acute $\mathrm{ON}$ in an eye with a history of $\mathrm{ON}$ were excluded. Recent or ongoing corticosteroid treatment was not an exclusion criterion.

\section{Standard Protocol Approvals, Registrations, and Patient Consents}

This study (IRMANO; NCT03651362) was approved by our local ethical committee of Lille University Hospital. Written informed consent was obtained for all participants. Subjects' consent was obtained according to the Declaration of Helsinki.

\section{Data Acquisition and Analysis}

Each patient underwent a brain MRI examination at inclusion $\left(\mathrm{M}_{0}\right)$ and at 12 months $\left(\mathrm{M}_{12}\right)$. Brain MRI were performed on a 3-T MRI (Achieva; Philips, Best, The Netherlands) with the use of a 32-channel array head coil. Brain MRI protocol included a 3D-DIR MR sequence with the following parameters: sagittal acquisition, voxel size $(1.2 \times 1.2 \times 1.3 \mathrm{~mm})$, TR 5500, TE 252, TI Dual 625/2,600, NEX 2, fat suppression SPIR, matrix size $208 \times 208$, FOV $250 \times 250 \times 195$, number of slices 300 , and sense 2 . During the 3D-DIR acquisition, patients were asked to close their eyes and avoid eye movements as much as possible. Signal abnormality was defined as increased signal intensity within the optic nerve compared with the normal white matter signal intensity. To exclude partial volume contamination and to avoid uncertainty on the differentiation of the nerve from the meningeal sheath, optic nerve DIR hypersignal was identified on multiple planes (Figure 1, A-C). Detection of demyelinating lesions on optic nerve/chiasma/optic tracts was performed by a consensus reading of 3D-DIR sequences by 2 trained investigators (M.D. and O.O.) who were blinded to clinical and OCT data. The presence/absence of
DIR hypersignal was recorded for not only each symptomatic nerve but also asymptomatic optic nerves. We defined 5 lesion locations: orbital, canalicular, cisternal, chiasmal, and optic tract. MRI at inclusion and MRI at $M_{12}$ were analyzed independently. Length of optic nerve DIR hypersignal was manually measured directly on MRI workstation. ${ }^{10}$ If more than 1 hypersignal was present on 1 nerve, length was defined as the sum of the length of each hypersignal regardless of the presence of gadolinium enhancement.

The group of eyes with acute $\mathrm{ON}$ will be considered as eyes with acute $\mathrm{ON}$ group. Fellow eyes will be categorized into 3 other groups: an eye group that already presented an $\mathrm{ON}$ (eyes with a history of $\mathrm{ON}$ ), another eye group without a history of $\mathrm{ON}$ but with asymptomatic optic nerve lesion (eyes with asymptomatic optic nerve lesion), and another group without a history of $\mathrm{ON}$ and without asymptomatic optic nerve lesion (eyes without optic nerve lesion).

OCT examination with a spectral Domain-OCT (Spectralis, Heidelberg Engineering, Germany) was conducted on the same day as MRI (inclusion and $\mathrm{M}_{12}$ ) and at $\mathrm{M}_{3}$ also. Our OCT protocol included a peripapillary scan for measuring peripapillary retinal nerve fiber layer (pRNFL; $12^{\circ}, 3.4 \mathrm{~mm}$ circular scan around the optic nerve with a minimum of 50 automatic real time) respecting OSCAR-IB criteria ${ }^{26}$ and a macular scan consisting of 25 vertical scans centered on the fovea (a minimum of 25 automatic real time). A macular segmentation was performed with HEYEX software (multilayer segmentation algorithm, Heidelberg Engineering, version 6.0.0.3). The mean volume (Early Treatment Diabetic Retinopathy Study $6 \mathrm{~mm}$ disc) was calculated for the macular ganglion cells layer coupled to macular inner plexiform layer (GCIPL) and for the inner nuclear layer (INL). Best-corrected 
Table 1 Demographics of the Whole Cohort and Different Patients' Subgroups

\begin{tabular}{|c|c|c|c|c|c|}
\hline & \multirow{2}{*}{$\begin{array}{l}\text { Whole cohort } \\
\begin{array}{l}\text { All } \\
(n=51)\end{array}\end{array}$} & \multicolumn{4}{|c|}{ Patients' subgroups } \\
\hline & & $\begin{array}{l}\text { CIS } \\
(n=23)\end{array}$ & $\begin{array}{l}\text { RRMS } \\
(n=20)\end{array}$ & $\begin{array}{l}\text { NMOSD } \\
(n=2)\end{array}$ & $\begin{array}{l}\text { Isolated ON } \\
(n=6)\end{array}$ \\
\hline $\operatorname{Sex}(F / M)$ & $33 / 18$ & $17 / 6$ & $10 / 10$ & $2 / 0$ & $4 / 2$ \\
\hline Age & $32.43 \pm 7.89$ & $33.43 \pm 8.10$ & $31.20 \pm 7.44$ & $35.50 \pm 15.59$ & $31.67 \pm 5.25$ \\
\hline Mean $( \pm S D)$ disease duration in mo & $20.94 \pm 54.04$ & $0.35 \pm 0.48$ & $50.30 \pm 77.36$ & $0.50 \pm 0.58$ & $8.83 \pm 19.23$ \\
\hline Number of patients with a history of ON (\%) & $5(9.8 \%)$ & $0(0 \%)$ & $4(10 \%)$ & $0(0 \%)$ & $1(8.3 \%)$ \\
\hline Mean $( \pm$ SD) delay between first visual symptoms and inclusion (in wk) & $2.80 \pm 1.99$ & $2.04 \pm 1.44$ & $3.65 \pm 2.08$ & $5.00 \pm 3.46$ & $2.17 \pm 1.40$ \\
\hline Number of patients under fingolimod at inclusion (\%) & $2(3.9 \%)$ & $0(0 \%)$ & $2(5 \%)$ & $0(0 \%)$ & $0(0 \%)$ \\
\hline Number of patients under fingolimod at M12 (\%) & $5(9.8 \%)$ & $0(0 \%)$ & $5(25 \%)$ & $0(0 \%)$ & $0(0 \%)$ \\
\hline
\end{tabular}

Abbreviations: CIS = clinically isolated syndrome; NMOSD = neuromyelitis optica spectrum disorders; ON = optic neuritis; RRMS = relapsing-remitting multiple sclerosis.

low-contrast monocular visual acuity (2.5\%; LCMVA) was measured with printed scales (PRECISION-VISION n²180) in LogMAR unit, at inclusion, $\mathrm{M}_{3}$, and $\mathrm{M}_{12}$.

\section{Statistical Analysis}

A sample size of 51 patients will have $80 \%$ power to detect a negative strong correlation between optic nerve lesion length at the acute phase of $\mathrm{ON}$ and retinal neuro-axonal loss at 12 months (H0: $r=-0.585$ vs $\mathrm{H} 1: \mathrm{r}=-0.8)^{10}$ with a 0.052 -sided significance level and taking into account $10 \%$ of nonanalyzable data.

Qualitative variables were reported as frequency (percentage) and continuous variables as mean $\pm \mathrm{SD}$ in case or normal distribution or median (first quartile [Q1]-third quartile [Q3]) otherwise. Normality was assessed graphically and using the Shapiro-Wilk test. Volume change of GCIPL was measured as a difference between value at $\mathrm{M}_{0}$ and value at $\mathrm{M}_{12}$. Because of clinical or subclinical papillary edema occurring at the acute phase of ON, thickness change of pRNFL was measured as a difference between value at $\mathrm{M}_{3}$ and value at $\mathrm{M}_{12}$. Volume change of INL was measured as a difference between value at $M_{0}$ and value at $M_{12}$.

Associations of length and topography of the optic nerve lesion assessed at the acute phase of ON with retinal neuro-axonal loss, retinal remodeling process, and visual impairment at 12 months (treated as dependent variables into models) were assessed using analysis of covariance (ANCOVA) adjusted for predefined confounders (retinal thickness/volume at baseline, delay between first visual symptoms and inclusion in the study, initiation of fingolimod treatment during the prospective 12month FU). Residual normality of ANCOVA models were checked by examining the Quantile-Quantile plots.

A comparison in length of optic nerve lesion length between inclusion and at 12 month assessments was performed using Wilcoxon signed rank test. Associations at the chronic stage $\left(\mathrm{M}_{12}\right)$ of optic nerve lesion length with retinal layer volumes (treated as dependent variables into models) were studied using univariable linear regression models. Residual normality of linear regression models were checked by examining the Quantile-Quantile plots. All statistical tests were conducted at the two-tailed $\alpha$ level of 0.05 using SAS software, release 9.4 (SAS Institute, Cary, NC).

\section{Data Availability}

The data that support the findings of this study are available from the corresponding author on reasonable request.

\section{Results}

\section{Population}

We recruited 51 patients presenting with an acute episode of $\mathrm{ON}$. Among them 23 patients exhibited a CIS suggestive of MS, 20 a clinically definite relapsing-remitting multiple sclerosis (RRMS), 2 a first event of NMOSD (seropositive for antiaquaporin 4 antibodies [Abs]) and 6 an isolated ON. No patient with isolated ON presented anti-MOG Abs. Four patients with RRMS and 1 patient with isolated $\mathrm{ON}$ had already presented a clinical ON episode of the fellow eye. One patient with RRMS presented a bilateral acute episode of $\mathrm{ON}$. This patient showed negative results for anti-aquaporin 4 and anti-MOG $\mathrm{Abs}$ and presented typical clinical and MRI features of MS. ${ }^{22}$ Demographic and clinical characteristics of our cohort are summarized in Table 1 .

In Table 2, we summarized the MRI, OCT, and contrast vision characteristics according to the different eyes' subgroups: eyes with acute $\mathrm{ON}(\mathrm{n}=52)$, eyes with a history of ON ( $=5)$, eyes with asymptomatic optic nerve lesion(s) $(n=7)$, and eyes without a history of ON and without optic nerve lesion $(\mathrm{n}=38)$. We found a symptomatic optic nerve DIR hypersignal at inclusion in all but $1 \mathrm{ON}$ eyes. This patient with RRMS patient was suspected of a first right $\mathrm{ON}$ episode, but optic nerve MRI at the acute phase or later did not detect any optic nerve lesion, and GCIPL thickness remained absolutely stable during the FU. At baseline, the mean optic nerve lesion length and retinal layers volume were comparable 
Table 2 MRI, OCT, and Visual Performance Characteristics of the Cohort and According to Different Eyes' Subgroups, at Inclusion and at 12 Months

\begin{tabular}{|c|c|c|c|c|c|c|c|c|c|c|c|}
\hline & & & $\mathbf{M}_{0}$ & & & $\mathbf{M}_{3}$ & & & $M_{1}$ & & \\
\hline & & & $\mathrm{n}$ & Mean \pm SD & Median (Q1-Q3) & $\mathbf{n}$ & Mean \pm SD & Median (Q1-Q3) & $\mathrm{n}$ & Mean \pm SD & Median (Q1-Q3) \\
\hline Eyes with acute ON $(n=52)$ & MRI & Length & 51 & $12.35 \pm 5.98$ & $11.20(8.85-14)$ & - & - & - & 50 & $15.76 \pm 8.70$ & $13.00(10.3-20)$ \\
\hline & OCT & GCIPL & 52 & $1.90 \pm 0.18$ & $1.91(1.78-2.015)$ & 43 & $1.69 \pm 0.20$ & $1.69(1.56-1.82)$ & 50 & $1.67 \pm 0.21$ & $1.68(1.54-1.81)$ \\
\hline & & pRNFL & 52 & $109.31 \pm 28.94$ & $102(93-115)$ & 43 & $87.64 \pm 13.82$ & $89(80.5-98)$ & 50 & $83.52 \pm 14.28$ & $87.5(75-93)$ \\
\hline & & INL & 52 & $0.96 \pm 0.07$ & $0.97(0.91-1.01)$ & 43 & $0.98 \pm 0.08$ & $0.97(0.91-1.04)$ & 50 & $0.98 \pm 0.08$ & $0.985(0.91-1.04)$ \\
\hline & Vision & LCMVA & 46 & $0.96 \pm 0.22$ & $1.1(0.8-1.1)$ & 43 & $0.78 \pm 0.23$ & $0.76(0.56-1.05)$ & 50 & $0.69 \pm 0.23$ & $0.62(0.50-0.80)$ \\
\hline Eyes with a history of ON $(n=5)$ & MRI & Length & 5 & $17.66 \pm 10.22$ & $14.8(14.8-25)$ & - & - & - & 5 & $14.74 \pm 6.66$ & $14.70(14.60-18.50)$ \\
\hline & OCT & GCIPL & 5 & $1.76 \pm 0.14$ & $1.76(1.74-1.77)$ & 5 & $1.77 \pm 0.15$ & $1.76(1.75-1.81)$ & 5 & $1.78 \pm 0.14$ & $1.77(1.73-1.81)$ \\
\hline & & pRNFL & 5 & $82.6 \pm 5.59$ & $84(83-86)$ & 5 & $82.00 \pm 6.04$ & $83(82-85)$ & 5 & $82.40 \pm 4.83$ & $84(83-85)$ \\
\hline & & INL & 5 & $1.01 \pm 0.13$ & $0.96(0.94-0.98)$ & 5 & $0.99 \pm 0.13$ & $0.93(0.93-0.98)$ & 5 & $0.98 \pm 0.12$ & $0.93(0.92-0.99)$ \\
\hline & Vision & LCMVA & 5 & $0.66 \pm 0.26$ & $0.60(0.58-0.64)$ & 5 & $0.57 \pm 0.18$ & $0.66(0.50-0.66)$ & 5 & $0.47 \pm 0.04$ & $0.50(0.44-0.50)$ \\
\hline Eyes with asymptomatic optic & MRI & Length & 7 & $8.00 \pm 2.31$ & $8.1(7-9.4)$ & - & - & - & 7 & $7.90 \pm 2.63$ & $7.30(6.95-9.55)$ \\
\hline & OCT & GCIPL & 7 & $1.98 \pm 0.16$ & $2.01(1.86-2.09)$ & 5 & $2.03 \pm 0.14$ & $2.04(1.98-2.11)$ & 7 & $1.95 \pm 0.15$ & $1.90(1.85-2.05)$ \\
\hline & & pRNFL & 7 & $96.14 \pm 3.76$ & $97(93.50-98.50)$ & 5 & $95.20 \pm 3.27$ & $96(95-96)$ & 7 & $94.71 \pm 3.55$ & $95(92-97)$ \\
\hline & & INL & 7 & $0.99 \pm 0.07$ & $0.99(0.95-1.03)$ & 5 & $0.97 \pm 0.06$ & $0.97(0.95-1.02)$ & 7 & $0.98 \pm 0.05$ & $0.98(0.96-1.02)$ \\
\hline & Vision & LCMVA & 7 & $0.47 \pm 0.08$ & $0.50(0.43-0.53)$ & 5 & $0.59 \pm 0.12$ & $0.56(0.50-0.68)$ & 7 & $0.59 \pm 0.12$ & $0.54(0.50-0.69)$ \\
\hline Eyes without a history of ON and & MRI & Length & - & - & - & - & - & - & - & - & - \\
\hline & OCT & GCIPL & 38 & $1.93 \pm 0.13$ & $1.92(1.84-2.03)$ & 32 & $1.92 \pm 0.14$ & $1.89(1.81-2.04)$ & 36 & $1.93 \pm 0.15$ & $1.9(1.86-2.05)$ \\
\hline & & pRNFL & 38 & $98.24 \pm 11.87$ & $98.5(91.25-107.75)$ & 32 & $96.78 \pm 11.15$ & $98.50(91.75-101.75)$ & 36 & $98.14 \pm 12.00$ & $98.5(90-105)$ \\
\hline & & INL & 38 & $0.94 \pm 0.06$ & $0.93(0.9-0.97)$ & 32 & $0.94 \pm 0.06$ & $0.93(0.91-0.98)$ & 36 & $0.95 \pm 0.06$ & $0.98(0.96-1.02)$ \\
\hline & Vision & LCMVA & 34 & $0.57 \pm 0.16$ & $0.57(0.46-0.69)$ & 32 & $0.58 \pm 0.14$ & $0.54(0.50-0.685)$ & 36 & $0.56 \pm 0.16$ & $0.54(0.50-0.69)$ \\
\hline
\end{tabular}

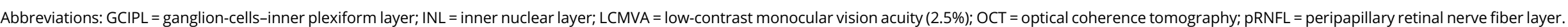
Values are reported as mean \pm SD and median (first quartile [Q1]-third quartile [Q3]). LCMVAs (2.5\%) are indicated in LogMAR unit. 
Figure 2 Flowchart of IRMANO Cohort and the Corresponding Eyes' Subgroups According to the Occurrence of an Acute ON, ON History, and Optic Nerve MRI Data

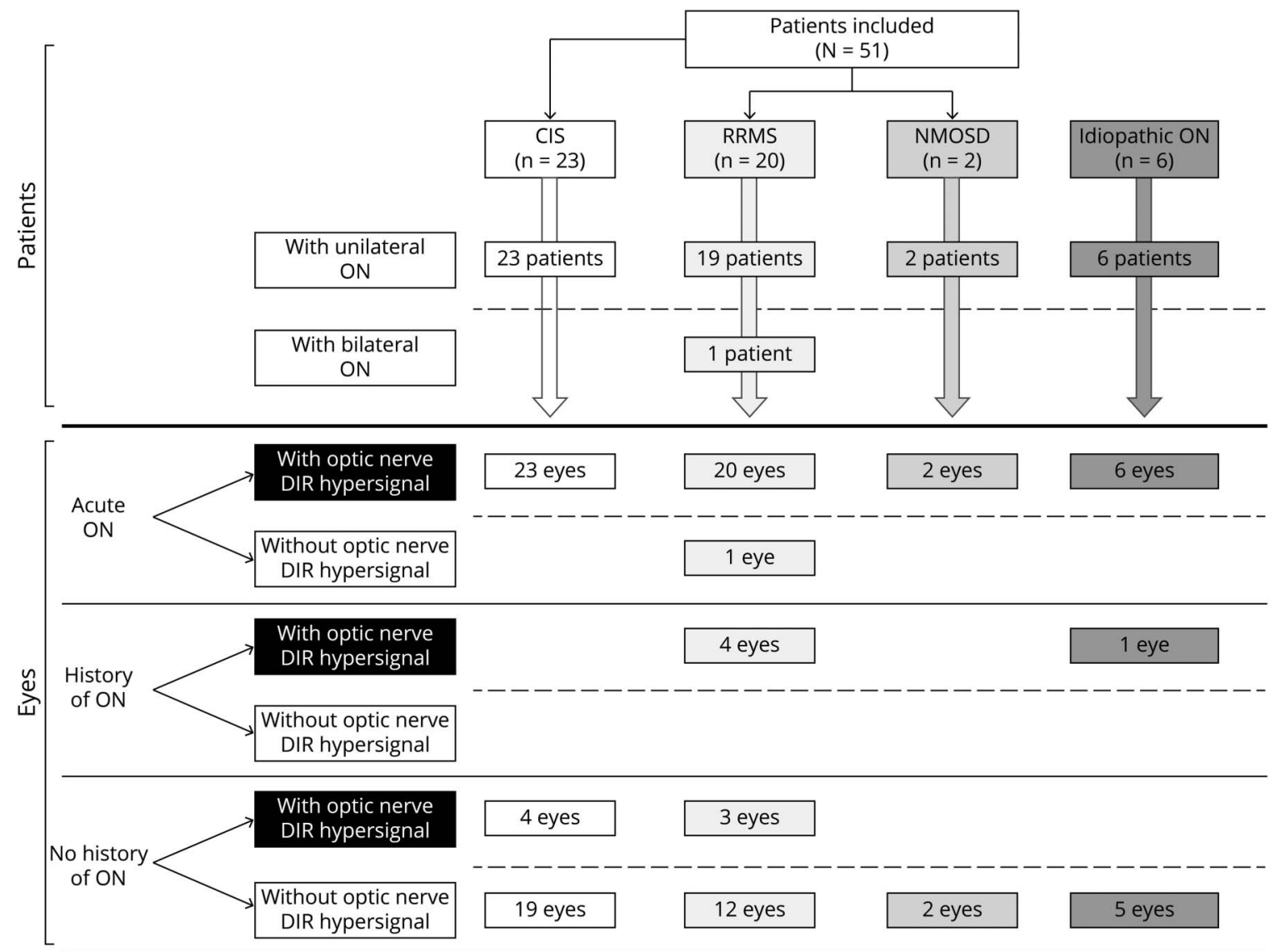

The authors recruited 51 patients presenting an acute episode of ON. One patient with acute ON did not present any optic nerve lesion on MRI. One patient with RRMS presented a bilateral acute ON. All eyes with a history of ON presented an optic nerve lesion. Some patients with CIS and RRMS presented an asymptomatic optic nerve lesion on the fellow eye. CIS = clinically isolated syndrome; DIR = double-inversion recovery; NMOSD = neuromyelitis optica spectrum disorders; ON = optic neuritis; RRMS = relapsing-remitting multiple sclerosis.

between CIS-RRMS and NMOSD-ION groups (eTable 1, links.lww.com/NXI/A696).

Gadolinium enhancement was observed in 33 eyes presenting acute ON (63.5\%). We found an optic nerve DIR hypersignal in all the 5 eyes with a history of ON (4 RRMS and 1 ION). Among the 45 eyes without a history of $\mathrm{ON}$, we found an asymptomatic optic nerve DIR hypersignal in 7 cases (4 patients with CIS and 3 with RRMS). All optic nerve lesions detected at inclusion were also observed at $\mathrm{M}_{12}$. The flowchart of our cohort is reported on Figure 2. At baseline, we detected 2 optic nerve lesions among 7 ON eyes (4 patients with CIS and 3 with RRMS). The length of these 2 lesions was added. No patient presented any new clinical episode of $\mathrm{ON}$ during the 12-month FU period. We did not observe any microcystic macular edema (MME) at baseline or during the FU period on OCT.

One patient with CIS presented a novel asymptomatic right optic nerve lesion at 12 months (Figure 3). GCIPL volume of this eye without optic nerve lesion at inclusion slightly decreased between inclusion $\left(1.92 \mathrm{~mm}^{3}\right)$ and $\mathrm{M}_{12}\left(1.88 \mathrm{~mm}^{3}\right)$.
Among all patients, 5 patients with MS (9.8\%) were under a DMT. Among them, 2 were treated by beta interferon, 2 by glatiramer acetate, and 1 by fingolimod for more than 1 year. Four patients initiated fingolimod during the study. None of these patients presented any macular edema. All patients received corticosteroid infusions before or at the time of their inclusion. All patients with NMOSD were additionally treated with plasmatic exchanges. Two patients ( 1 patient with RRMS and 1 with CIS) were lost on FU at $\mathrm{M}_{12}$.

\section{Association Between Optic Nerve Lesion Length at the Acute Phase of Optic Neuritis and Retinal Changes and Chronic Visual Impairment}

We found significant association between optic nerve lesion length measured at inclusion and GCIPL volumes decrease between inclusion and $\mathrm{M}_{12}$ (estimate \pm standard error; $-0.012 \pm 0.004 ; p=0.0016$ ) (Table 3 ). This means that each millimeter of optic nerve lesion length measured at the acute phase of ON is associated with a GCIPL volume decrease of $0.012 \mathrm{~mm}^{3}$ at the 12 -month FU. We also found significant association between optic nerve lesion length and INL 

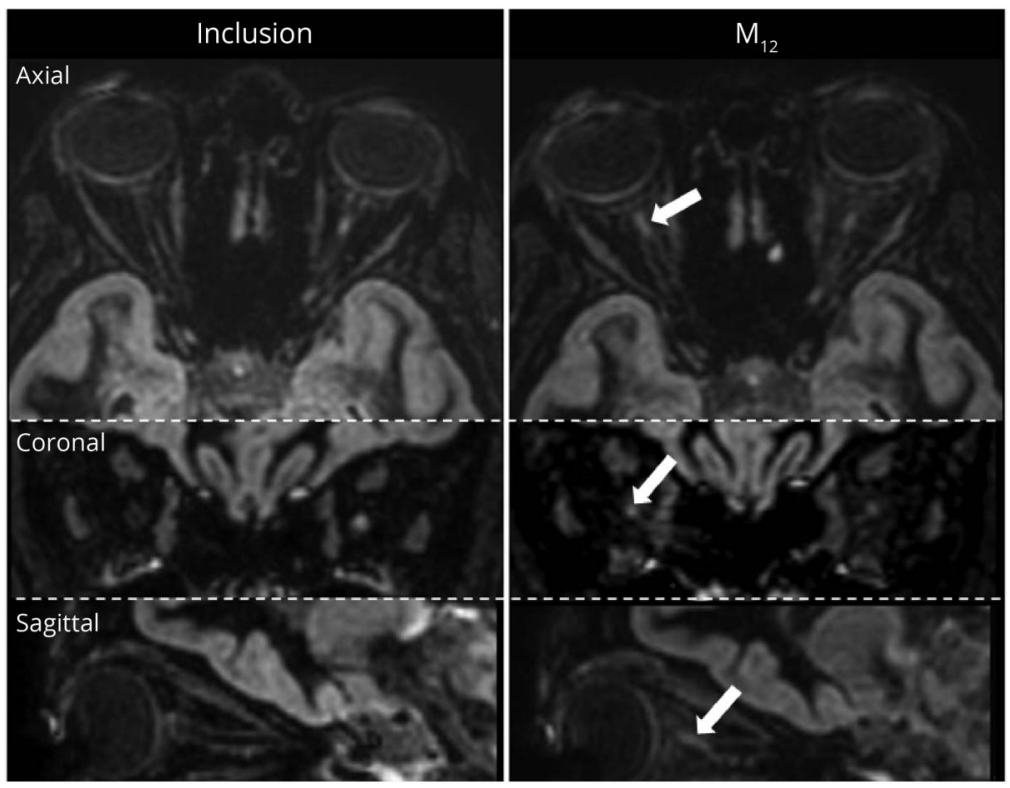

Optic nerve MRI of a patient with CIS presenting a left acute optic neuritis. At inclusion, no lesion was detected on the right optic nerve. At $\mathrm{M}_{12}$, a new right asymptomatic retrobulbar optic nerve DIR hypersignal was detected (white arrows). CIS = clinically isolated syndrome.

volume increase between inclusion and $\mathrm{M}_{12}$ or LCMVA measured at $\mathrm{M}_{12}$. We did not find any association between optic nerve lesion length at inclusion and pRNFL decrease. If we focused only on patients experiencing RRMS or CIS, we found comparable results. We observed a trend toward significance between optic nerve lesion length and INL thickening. Results are summarized in Table 3.

\section{Association Between Optic Nerve Lesion Location at the Acute Phase and Retinal Neuro- Axonal Loss and Visual Impairment}

Involvement of the different parts of the optic nerve in the whole cohort and different eyes' subgroups is summarized in Table 4. Among acute ON eyes, orbital involvement was observed in 36 patients (69.2\%), canalicular involvement in 21 patients $(40.4 \%)$, cisternal involvement in 13 patients $(25 \%)$, and chiasmal involvement in 2 patients (3.8\%). We did not report any involvement of the optic tracts. No association was observed between any optic nerve lesion site and the GCIPL thinning. However, we found significant association between cisternal optic nerve involvement and a greater pRNFL thinning $\left(\mathrm{M}_{3}-\mathrm{M}_{12} ; p=0.034\right)$. Indeed, we found significant association between involvement of the orbital part at inclusion and a lower visual impairment at $\mathrm{M}_{12}(p=0.027)$. At the opposite, we found significant association between involvement of the cisternal part at inclusion and higher visual impairment at $M_{12}(p=0.014)$. No association was observed for any other location (canalicular, chiasma, and optic tracts).

\section{Evolution of Optic Nerve Lesion Length}

Considering eyes with acute $\mathrm{ON}$, the mean optic nerve lesion length significantly increased between inclusion $(12.35 \pm 5.98)$ and $\mathrm{M}_{12}(15.76 \pm 8.70 ; p=0.0007)$. The increase in optic nerve lesion length was significantly associated with the GCIPL thinning between inclusion and $\mathrm{M}_{12}(-0.012 \pm 0.003 ; p=0.0011)$.

Considering eyes with asymptomatic optic nerve lesion and eyes with a history of ON together or separately, the optic nerve lesion length was stable between inclusion and $\mathrm{M}_{12}(p=$ 0.25 for together, $p=0.61$ for asymptomatic optic nerve lesion, and $p=0.31$ for eyes with a history of $\mathrm{ON}$ ).

\section{Association Between Optic Nerve Lesion Length and Retinal Layers Volumes at the Chronic Stage $\left(\mathrm{M}_{12}\right)$}

We observed significant association between the optic nerve lesion length measured at $M_{12}$ and retinal layer volume at $M_{12}$ or chronic visual impairment. The higher was the extent of optic nerve lesion, the higher were the retinal neuro-axonal loss, the retinal remodeling, and the chronic visual impairment. Results are close to our previous studies and summarized in eTable 2, links.lww.com/NXI/A696.

\section{Classification of Evidence}

This study provides Class I evidence that optic nerve lesion length measured on MRI during the acute phase of a first episode of $\mathrm{ON}$ is associated with long-term retinal neuroaxonal loss and visual impairment.

\section{Discussion}

We found that optic nerve lesion length measured at the acute phase of $\mathrm{ON}$ is a predictive biomarker of retinal neuro-axonal loss and visual impairment at a chronic stage. We also showed that optic nerve lesion length at the acute phase is associated with retinal remodeling process (INL thickening) and that it 
Table 3 Associations Between ON Lesion Length Measured at the Acute Phase and Retinal Layer Changes Between Inclusion and at 12 Months or Chronic Visual Impairment at 12 months

\begin{tabular}{|c|c|c|c|c|c|c|}
\hline & \multicolumn{3}{|c|}{ Whole acute ON eyes cohort $(n=52)$} & \multicolumn{3}{|c|}{ Acute ON eyes cohort of CIS and MS $(n=44)$} \\
\hline & Estimate & Standard error & $p$ Value & Estimate & Standard error & $p$ Value \\
\hline GCIPL volume change $\left(\mathrm{mm}^{3}\right)$ & -0.012 & 0.004 & 0.0016 & -0.013 & 0.004 & 0.0011 \\
\hline pRNFL thickness change $(\mu \mathrm{m})$ at $\mathrm{M}_{12}$ & +0.036 & 0.156 & 0.82 & -0.024 & 0.152 & 0.87 \\
\hline INL volume change $\left(\mathrm{mm}^{3}\right)$ & +0.002 & 0.001 & 0.0263 & +0.002 & 0.001 & 0.0846 \\
\hline LCMVA $2.5 \%$ at M12 & +0.016 & 0.003 & $<0.0001$ & +0.014 & 0.005 & 0.0056 \\
\hline
\end{tabular}

Abbreviations: CIS = clinically isolated syndrome; GCIPL = ganglion-cells-inner plexiform layer; INL = inner nuclear layer; LCMVA = low-contrast monocular vision acuity (2.5\%); MS = multiple sclerosis; ON = optic neuritis; pRNFL = peripapillary retinal nerve fiber layer.

LCMVA (2.5\%) are indicated in LogMAR unit.

Significant $p$ values $(\geq 0.05)$ are indicated in bold.

slightly increased during the FU. Furthermore, the increase in optic nerve lesion length during the FU was associated with a greater retinal neuro-axonal loss and visual impairment.

We showed significant association between optic nerve lesion length at the acute phase and both structural and functional visual parameters. Thus, we clearly suggest that the optic nerve lesion length in acute $\mathrm{ON}$ can be considered as a predictive biomarker of retinal neuro-axonal loss, retinal remodeling process, and visual impairment. The greater is the optic nerve lesion length, the higher is the GCIPL thinning, INL thickening, and chronic visual impairment. After an ON episode, INL thickening is classical and may, in most severe cases, be associated with the presence of MME. MME is reported in nearly $4 \%$ of patients with RRMS and $20 \%$ of patients with NMOSD. ${ }^{27}$ Similar to others, ${ }^{18-20,28}$ we did not observe any association between optic nerve lesion length and pRNFL thinning. Large to very subtle optic nerve swelling is frequently observed at the acute stage of ON. ${ }^{29}$ This edema represents a confusing factor that affects and artificially increases the measurement of pRNFL thickness at the acute stage. For this reason, we have planned to measure pRNFL at $\mathrm{M}_{3}$ when papillary edema has disappeared and to evaluate pRNFL atrophy between $\mathrm{M}_{3}$ and $\mathrm{M}_{12}$. However, it could be possible that $\mathrm{M}_{3}$ evaluation was already too late to properly assess the pRNFL thinning. GCIPL is not affected by optic nerve inflammation and optic nerve edema and is probably a more accurate and relevant retinal imaging biomarker than pRNFL in neuroinflammatory disorders affecting the optic nerve. GCIPL intereye retinal thickness difference (IETD) is a better marker of asymptomatic optic nerve lesion in CIS than pRNFL IETD, ${ }^{30}$ and GCIPL thickness is better correlated to visual impairment than pRNFL. ${ }^{31}$ GCIPL thinning after a clinical episode of $\mathrm{ON}$ is an early event. Major part of the GCIPL thinning is observed during the first 2 months, and GCIPL thinning during the first month is predictive of shortterm (6 months) visual impairment. ${ }^{32}$ Despite the value of such a measurement, this early thinning of GCIPL is not strictly applicable at the acute stage of diagnosis as a predictive biomarker because it requires a 1-month FU OCT to predict remote chronic visual impairment.

We did not confirm that canalicular optic nerve involvement was associated with negative visual outcome, ${ }^{10,12,13,28}$ but our results suggest that cisternal involvement might be associated with negative visual outcome (higher pRNFL thinning and higher residual visual impairment) and that orbital optic nerve involvement might be associated with lower residual visual impairment. However, such results were not confirmed by GCIPL analysis. Because GCIPL has been shown to be a more

Table 4 Involvement of the Different Parts of the Optic Nerve in the Whole Cohort and Different Eyes' Subgroups

\begin{tabular}{llllll}
\hline & \multicolumn{4}{l}{ Involvement of the different parts of the optic nerve, $\mathbf{n}(\%)$} \\
\cline { 2 - 6 } & Orbital & Canalicular & Cisternal & Chiasmatic & Optic tract \\
\hline All eyes $(\mathbf{n}=\mathbf{1 0 2})$ & $47(46.1)$ & $25(24.5)$ & $16(15.7)$ & $2(2)$ & $0(0)$ \\
\hline Eyes with acute $\mathbf{O N}(\mathbf{n}=\mathbf{5 2})$ & $36(69.2)$ & $21(40.4)$ & $13(25)$ & $2(3.8)$ & $0(0)$ \\
\hline Eyes with a history of ON $(\mathbf{n}=\mathbf{5})$ & $5(100)$ & $2(40.0)$ & $2(40.0)$ & $0(0)$ & $0(0)$ \\
\hline Eyes with asymptomatic optic nerve lesions $(\mathbf{n}=\mathbf{7})$ & $6(85.7)$ & $2(28.6)$ & $1(14.3)$ & $0(0)$ & $0(0)$ \\
\hline Eyes without a history of ON and without optic nerve lesions $(\mathbf{n}=\mathbf{3 8})$ & $0(0)$ & $0(0)$ & $0(0)$ & $0(0)$ & $0(0)$ \\
\hline
\end{tabular}

Abbreviations: $\mathrm{ON}=$ optic neuritis. 
interesting and reliable OCT outcome parameter than pRNFL and because $p$ values remain close to the statistical threshold, cautiousness is needed when interpreting these results.

If some studies reported that optic nerve lesion length was stable between the acute and chronic phases, ${ }^{20,21}$ others reported that optic nerve lesion length increases with time..$^{13,14,16,33}$ One study has suggested that shortest lesions at the acute phase increased and longest lesions at the acute phase may decrease. ${ }^{34}$ In our cohort, we showed that the length of acute optic nerve lesion significantly increased and that the length of chronic symptomatic or asymptomatic optic nerve lesions was stable. Indeed, we observed that the increase in optic nerve lesion length was significantly associated with the subsequent GCIPL thinning in eyes with acute ON. If at the acute stage of ON, we may associate optic nerve DIR hypersignal with demyelination, our results suggest that the increasing in optic nerve lesion length is at least partly associated with the neurodegenerative process along the optic nerve after an $\mathrm{ON}$ episode. This process is bidirectional: anterograde (from the optic nerve lesion to the ipsilateral lateral geniculate nucleus) and retrograde (from the optic nerve lesion to the retina). Indeed, optic nerve DIR hypersignal can be observed in noninflammatory diseases, such as chronic glaucoma ${ }^{35}$ and Leber hereditary optic neuropathy. ${ }^{36}$ If detected at distance from acute $\mathrm{ON}$ or observed on a non-ON eye (NON eye), the extent of optic nerve lesion on MRI should probably be considered as the consequence of a dual process including demyelination and neurodegeneration (axonal loss). According to our results, a progression of the optic nerve lesion length could be useful to assess the efficacy of future neuroprotective drugs in acute $\mathrm{ON}$.

With this new independent prospective cohort, we replicated the previous results of our group showing that optic nerve lesion length at the chronic stage was correlated with the retinal layers volume/thickness and the chronic visual impairment. ${ }^{10}$ Thus, optic nerve lesion length is both a predictive biomarker of retinal axonal loss when measured at the acute phase of $\mathrm{ON}$ and a biomarker of retinal neuro-axonal loss if measured at a chronic stage of $\mathrm{ON}$ or in asymptomatic cases.

We did not observe optic nerve gadolinium enhancement in all affected optic nerves, but some patients might have already received corticosteroids before inclusion, especially in patients with known RRMS. Recent corticosteroid treatment was not an exclusion criterion in our study. In fact, previous studies have shown that corticosteroids did not affect optic nerve lesion length at the acute phase. ${ }^{3,34}$ Furthermore, length of optic nerve enhancement seems not different between patients with or without corticosteroids. ${ }^{28}$ In addition, some symptomatic optic nerve lesions were quite short, making more difficult detection of gadolinium enhancement.

In neuroinflammatory diseases and more particularly in MS, neurodegenerative process along the optic pathways is considered to be transsynaptic with an anterograde (from the retina to the cortex) and retrograde (from the cortex to the retina) manner and to be the consequence of demyelinating lesions on optic nerves, chiasma, optic tracts, and optic radiations. ${ }^{11}$ In our analysis, we did not adjust to the T2 lesion burden within the optic radiations because previous works from our group suggested the lack of association between optic radiation lesion volume and retinal layer volumes in $\mathrm{CIS}^{9}$ and RRMS. ${ }^{11}$ In addition, we included patients presenting with ION and NMOSD who did not present any significant asymptomatic $\mathrm{T} 2$ brain lesions.

In this study, we confirmed that 3D-DIR MRI sequence is sensitive for the detection of demyelinating optic nerve lesions by detecting all but 1 acute symptomatic optic nerve lesions. This suspected case of acute ON without optic nerve DIR hypersignal and GCPIL thinning might ultimately be considered a posteriori as an overdiagnosis of ON. If patients with MS referred to Neuro-Ophthalmologic unit for acute $\mathrm{ON}$ are more likely to experience ON, up to $4 \%$ may present an overdiagnosis of $\mathrm{ON}^{37}$ In this study, we showed again that asymptomatic optic nerve lesions are frequent in CIS and RRMS. Conversely, and as previously reported, ${ }^{10}$ this was not observed in patients with ION or NMOSD from our cohort. Detection of asymptomatic optic nerve lesion might be helpful both for the positive diagnosis of MS (i.e., future MS diagnosis criteria) and for differential diagnosis. Its presence may be supportive of MS diagnosis. In this study, evolution of the length of symptomatic and asymptomatic optic nerve lesions differs, suggesting that asymptomatic lesions are stable and older than symptomatic ones. Thus, we suggest that the presence of asymptomatic optic nerve lesions in CIS/RRMS might be representative of dissemination of disease in time ${ }^{38}$ and it might also be considered as a marker of dissemination in space. ${ }^{39,40}$

Our study has some limitations. First, we did not perform a complete neuro-ophthalmologic examination at $\mathrm{M}_{12}$ and evaluated only chronic visual impairment with a low-contrast visual acuity chart. Regardless of the study protocol, a complete neuro-ophthalmologic examination had been performed at 3-4 months. Post hoc complementary analysis is warranted. Second, multiple optic nerve lesions were detected on some symptomatic eyes, and we cannot exclude that such optic nerves may cumulate both symptomatic and asymptomatic lesions, especially in patients with CIS and RRMS. We acknowledge that it may represent a bias in our analysis, but these patients with multiple but unilateral optic nerve lesions are representative of the real-life practice. On the whole, onethird of patients with CIS/RRMS experienced asymptomatic optic nerve lesions. ${ }^{9,11}$ Indeed, presence or absence of gadolinium enhancement is not sufficient to distinguish a recent active lesion from a chronic one. Excluding eyes with multiple lesions from our analysis would have reduced the power of our statistical analysis. We preferred to stick to real-life practicing and to consider the worse-case scenario by adding the different lengths. Third, our cohort may seem as quite heterogeneous because we included patients with various causes of ON and did not focus only on cases with CIS and MS. 
However, OCT and MRI data of ON eyes at baseline were similar between CIS-RRMS and NMOSD-ION subgroups, and our results remained comparable when focusing only on CIS and RRMS. Finally, we could not exclude false-positive findings regarding multiple comparisons, but our study is exploratory, and we cannot certify that each symptomatic or asymptomatic optic nerve hypersignal corresponds to a demyelinating lesion and not to a lesion of another type.

Optic nerve MRI still remains a challenge, and interscanner variability remains an additional difficulty in applying optic nerve lesion length as an imaging biomarker, but sensitive optic nerve MRI sequence for detecting demyelinating lesion in routine are more and more developing. If it seems better to perform a 3D MRI sequence for measuring optic nerve lesion length, this remains feasible and reliable on a coronal 2D MRI sequence. Optic nerve MRI is important for the diagnosis of $\mathrm{ON}$, helpful for identifying the underlying demyelinating disease, ${ }^{2}$ and provides a promising imaging biomarker able to predict visual impairment and retinal changes at the acute phase of ON. The optic nerve lesion length should be taken into account in future algorithms for therapeutic management of acute $\mathrm{ON}$.

\section{Acknowledgment}

The authors thank Miss Julie Petit for her help and technical support and Maxime Thoor and Chloé Crinquette for the MRI acquisitions. The authors also thank Fondation VISIO for their constant support.

\section{Study Funding}

Fondation VISIO (Irmano grant).

\section{Disclosure}

The authors report no disclosures. Go to Neurology.org/NN for full disclosures.

\section{Publication History}

Received by Neurology: Neuroimmunology \& Neuroinflammation September 10, 2021. Accepted in final form December 3, 2021.

\section{Appendix Authors}

\begin{tabular}{lll}
\hline Name & Location & Contribution \\
\hline $\begin{array}{l}\text { Mickael Denis, } \\
\text { MD }\end{array}$ & $\begin{array}{l}\text { University of } \\
\text { Lille, France }\end{array}$ & $\begin{array}{l}\text { Analysis and interpretation of the data; } \\
\text { drafting and revising the article for } \\
\text { intellectual content }\end{array}$ \\
\hline $\begin{array}{l}\text { Jean-Philippe } \\
\text { Woillez, MD, } \\
\text { PhD }\end{array}$ & $\begin{array}{l}\text { University of } \\
\text { Lille, France }\end{array}$ & Major role in the acquisition of data \\
\hline $\begin{array}{l}\text { Vasily M. } \\
\text { Smirnov, MD }\end{array}$ & $\begin{array}{l}\text { University of } \\
\text { Lille, France }\end{array}$ & $\begin{array}{l}\text { Analysis and interpretation of the data; } \\
\text { drafting and revising the article for } \\
\text { intellectual content }\end{array}$ \\
\hline Elodie Drumez & $\begin{array}{ll}\text { University of } \\
\text { Lille, France }\end{array}$ & $\begin{array}{l}\text { Design and conceptualization of the } \\
\text { study; } \\
\text { analysis of the data }\end{array}$ \\
& &
\end{tabular}

\section{Appendix (continued)}

\begin{tabular}{|c|c|c|}
\hline Name & Location & Contribution \\
\hline $\begin{array}{l}\text { Julien Lannoy, } \\
\text { MD }\end{array}$ & $\begin{array}{l}\text { University of } \\
\text { Lille, France }\end{array}$ & $\begin{array}{l}\text { Revising the article for intellectual } \\
\text { content }\end{array}$ \\
\hline $\begin{array}{l}\text { Julie Boucher, } \\
\text { MD }\end{array}$ & $\begin{array}{l}\text { University of } \\
\text { Lille, France }\end{array}$ & $\begin{array}{l}\text { Revising the article for intellectual } \\
\text { content }\end{array}$ \\
\hline $\begin{array}{l}\text { Mickael Zedet, } \\
\text { MD }\end{array}$ & $\begin{array}{l}\text { University of } \\
\text { Lille, France }\end{array}$ & $\begin{array}{l}\text { Revising the article for intellectual } \\
\text { content }\end{array}$ \\
\hline $\begin{array}{l}\text { Jean-Pierre } \\
\text { Pruvo, MD, PhD }\end{array}$ & $\begin{array}{l}\text { University of } \\
\text { Lille, France }\end{array}$ & $\begin{array}{l}\text { Revising the article for intellectual } \\
\text { content }\end{array}$ \\
\hline $\begin{array}{l}\text { Julien } \\
\text { Labreuche }\end{array}$ & $\begin{array}{l}\text { University of } \\
\text { Lille, France }\end{array}$ & $\begin{array}{l}\text { Design and conceptualization of the } \\
\text { study; analysis of the data }\end{array}$ \\
\hline $\begin{array}{l}\text { Helene Zephir, } \\
\text { MD, PhD }\end{array}$ & $\begin{array}{l}\text { University of } \\
\text { Lille, France }\end{array}$ & $\begin{array}{l}\text { Analysis and interpretation of the data; } \\
\text { drafting and revising the article for } \\
\text { intellectual content }\end{array}$ \\
\hline $\begin{array}{l}\text { Xavier Leclerc, } \\
\text { MD, PhD }\end{array}$ & $\begin{array}{l}\text { University of } \\
\text { Lille, France }\end{array}$ & $\begin{array}{l}\text { Analysis and interpretation of the data; } \\
\text { drafting and revising the article for } \\
\text { intellectual content }\end{array}$ \\
\hline $\begin{array}{l}\text { Olivier } \\
\text { Outteryck, MD, } \\
\text { PhD }\end{array}$ & $\begin{array}{l}\text { University of } \\
\text { Lille, France }\end{array}$ & $\begin{array}{l}\text { Design and conceptualization of the } \\
\text { study; } \\
\text { acquisition of data; } \\
\text { analysis and interpretation of the data; } \\
\text { drafting and revising the article for } \\
\text { intellectual content; and } \\
\text { study supervision }\end{array}$ \\
\hline
\end{tabular}

\section{References}

1. Toosy AT, Mason DF, Miller DH. Optic neuritis. Lancet Neurol. 2014;13(1):83-99.

2. Petzold A, Wattjes MP, Costello F, et al. The investigation of acute optic neuritis: a review and proposed protocol. Nat Rev Neurol. 2014;10(8):447-458.

3. Akaishi $\mathrm{T}, \mathrm{T}$ akeshita $\mathrm{T}$, Himori $\mathrm{N}$, et al. Rapid administration of high-dose intravenous methylprednisolone improves visual outcomes after optic neuritis in patients with AQP4-IgG-positive NMOSD. Front Neurol. 2020;11:932.

4. Kleiter I, Gahlen A, Borisow N, et al. Neuromyelitis optica: evaluation of 871 attacks and 1,153 treatment courses. Ann Neurol. 2016;79(2):206-216.

5. Lubetzki C, Zalc B, Williams A, Stadelmann C, Stankoff B Remyelination in multiple sclerosis: from basic science to clinical translation. Lancet Neurol. 2020;19:678-688.

6. Kolappan M, Henderson AP, Jenkins TM, et al. Assessing structure and function of the afferent visual pathway in multiple sclerosis and associated optic neuritis. J Neurol. 2009;256(3): 305-319.

7. Hodel J, Outteryck O, Bocher AL, et al. Comparison of $3 \mathrm{D}$ double inversion recovery and 2D STIR FLAIR MR sequences for the imaging of optic neuritis: pilot study. Eur Radiol. 2014;24(12):3069-3075.

8. Sartoretti T, Sartoretti E, Rauch S, et al. How common is signal-intensity increase in optic nerve segments on $3 \mathrm{D}$ double inversion recovery sequences in visually asymptomatic patients with multiple sclerosis? AJNR Am J Neuroradiol. 2017;38 1748-1753.

9. London F, Zéphir H, Drumez E, et al. Optical coherence tomography: a window to the optic nerve in clinically isolated syndrome. Brain. 2019;142(4):903-915.

10. Hadhoum N, Hodel J, Defoort-Dhellemmes S, et al. Length of optic nerve double inversion recovery hypersignal is associated with retinal axonal loss. Mult Scler. 2016; 22(5):649-658.

11. Davion JB, Lopes R, Drumez É, et al. Asymptomatic optic nerve lesions: an underestimated cause of silent retinal atrophy in MS. Neurology. 2020;94(23):e2468-2478

12. Miller DH, Newton MR, van der Poel JC, et al. Magnetic resonance imaging of the optic nerve in optic neuritis. Neurology. 1988;38(2):175-179.

13. Dunker S, Wiegand W. Prognostic value of magnetic resonance imaging in monosymptomatic optic neuritis. Ophthalmology. 1996;103(11):1768-1773.

14. Tur C, Goodkin O, Altmann DR, et al. Longitudinal evidence for anterograde transsynaptic degeneration after optic neuritis. Brain. 2016;139(Pt 3):816-828.

15. Akaishi T, Nakashima I, Takeshita $\mathrm{T}$, et al. Lesion length of optic neuritis impacts visual prognosis in neuromyelitis optica. J Neuroimmunol. 2016;293:28-33.

16. Hickman SJ, Toosy AT, Miszkiel KA, et al. Visual recovery following acute optic neuritis, a clinical, electrophysiological and magnetic resonance study. J Neurol. 2004; 251:996-1005

17. Hickman SJ, Toosy AT, Jones SJ, et al. A serial MRI study following optic nerve mean area in acute optic neuritis. Brain. 2004;127(pt 11):2498-2505.

18. Pro MJ, Pons ME, Liebmann JM, et al. Imaging of the optic disc and retinal nerve fiber layer in acute optic neuritis. J Neurol Sci. 2006;250(1-2):114-119. 
19. Kupersmith MJ, Mandel G, Anderson S, Meltzer DE, Kardon R. Baseline, one and three month changes in the peripapillary retinal nerve fiber layer in acute optic neuritis: relation to baseline vision and MRI. J Neurol Sci. 2011;308(1-2):117-123.

20. Fuglø D, Kallenbach K, Tsakiri A, et al. Retinal atrophy correlates with fMRI response in patients with recovered optic neuritis. Neurology. 2011;77:645-651.

21. Zhang Y, Metz LM, Scott JN, Trufyn J, Fick GH, Costello F. MRI texture heterogeneity in the optic nerve predicts visual recovery after acute optic neuritis. Neuroimage Clin. 2014;4:302-307.

22. Thompson AJ, Banwell BL, Barkhof F, et al. Diagnosis of multiple sclerosis: 2017 revisions of the McDonald criteria. Lancet Neurol. 2018;17(2):162-173.

23. Wingerchuk DM, Banwell B, Bennett JL, et al. International consensus diagnostic criteria for neuromyelitis optica spectrum disorders. Neurology. 2015;85(2): 177-189.

24. Kleerekooper I, Trip SA, Plant GT, Petzold A. Expanding the phenotype of MOG antibody-associated disease (MOGAD): half a century of epilepsy and relapsing optic neuritis. J Neurol Neurosurg Psychiatry. 2021:92(3):340-342.

25. Nolan R, Gelfand JM, Green AJ. Fingolimod treatment in multiple sclerosis leads to increased macular volume. Neurology. 2013;80(2):139-144.

26. Schippling S, Balk LJ, Costello F, et al. Quality control for retinal OCT in multiple sclerosis: validation of the OSCAR-IB criteria. Mult Scler. 2015;21(2):163-170.

27. Outteryck O, Vermersch P. OCT findings in neuromyelitis optica spectrum disorders. In: Petzold A, ed. Optical Coherence Tomography in Multiple Sclerosis. Springer international Publishing; 2016:85-96.

28. Kupersmith MJ, Alban T, Zeiffer B, Lefton D. Contrast-enhanced MRI in acute optic neuritis: relationship to visual performance. Brain. 2002;125(pt 4):812-822.

29. Beck RW, Cleary PA, Anderson MM, et al. A randomized, controlled trial of corticosteroids in the treatment of acute optic neuritis. N Engl J Med. 1992;326: $581-588$.
30. Outteryck O, Lopes R, Drumez É, et al. Optical coherence tomography for detection of asymptomatic optic nerve lesions in clinically isolated syndrome. Neurology. 2020;95(6): e733-e744.

31. Saidha S, Syc SB, Durbin MK, et al. Visual dysfunction in multiple sclerosis correlates better with optical coherence tomography derived estimates of macular ganglion cell layer thickness than peripapillary retinal nerve fiber layer thickness. Mult Scler. 2011;17(12):1449-1463.

32. Gabilongo I, Martinez-Lapiscina EH, Fraga-Pumar E, et al. Dynamics of retinal injury after acute optic neuritis. Ann Neurol. 2015;77:517-528.

33. Jenkins TM, Toosy AT, Ciccarelli O, et al. Neuroplasticity predicts outcome of optic neuritis independent of tissue damage. Ann Neurol. 2010;67(1):99-113.

34. Kapoor R, Miller DH, Jones SJ, et al. Effects of intravenous methylprednisolone on outcome in MRI-based prognostic subgroups in acute optic neuritis. Neurology. 1998; 50(1):230-237.

35. Sartoretti T, Stürmer J, Sartoretti E, et al. Long segment 3D double inversion recovery (DIR) hypersignal on MRI in glaucomatous optic neuropathy. BMC Ophthalmol. 2019;19:258.

36. Blanc C, Heran F, Habas C, Bejot Y, Sahel J, Vignal-Clermont C. MRI of the optic nerves and chiasm in patients with leber hereditary optic neuropathy. J Neuroophthalmol. 2018;38(4): 434437.

37. Stunkel L, Kung NH, Wilson B, McClelland CM, Van Stavern GP. Incidence and causes of overdiagnosis of optic neuritis. JAMA Ophthalmol. 2018;136(1):76-81.

38. London F, Zéphir H, Hadhoum N, et al. Optic nerve double inversion recovery hypersignal in patients with clinically isolated syndrome is associated with asymptomatic gadolinium-enhanced lesion. Mult Scler. 2019;25(14):1888-1895.

39. Filippi M, Rocca MA, Ciccarelli O, et al. MRI criteria for the diagnosis of multiple sclerosis: MAGNIMS consensus guidelines. Lancet Neurol. 2016;15(3):292-303.

40. Vidal-Jordana A, Rovira A, Arrambide G, et al. Optic nerve topography in multiple sclerosis diagnosis: the utility of visual evoked potentials. Neurology. 2021;96: e482-e490. 


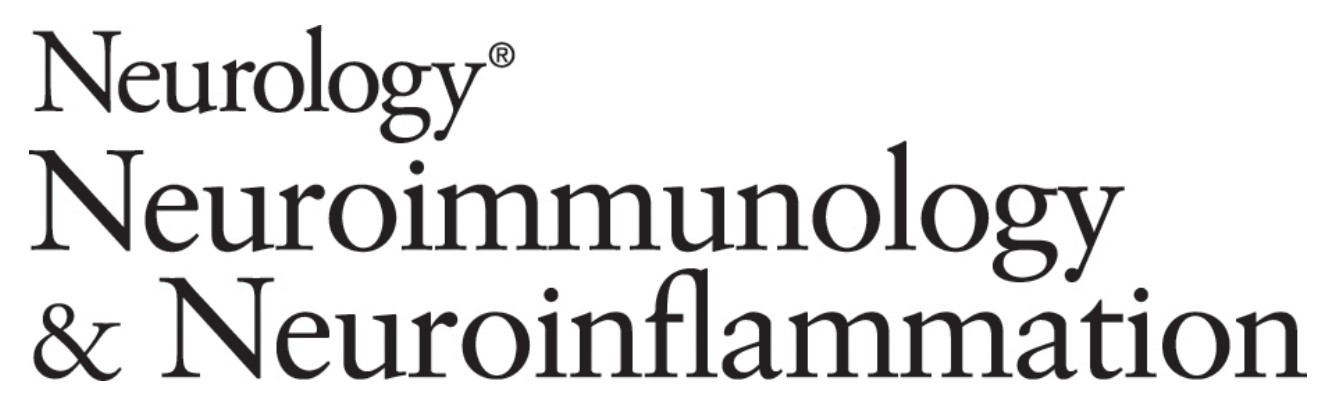

Optic Nerve Lesion Length at the Acute Phase of Optic Neuritis Is Predictive of Retinal Neuronal Loss

Mickael Denis, Jean-Philippe Woillez, Vasily M. Smirnov, et al.

Neurol Neuroimmunol Neuroinflamm 2022;9;

DOI 10.1212/NXI.0000000000001135

This information is current as of January 28, 2022

Neurol Neuroimmunol Neuroinflamm is an official journal of the American Academy of Neurology.

Published since April 2014, it is an open-access, online-only, continuous publication journal. Copyright

Copyright $\odot 2022$ The Author(s). Published by Wolters Kluwer Health, Inc. on behalf of the American

Academy of Neurology.. All rights reserved. Online ISSN: 2332-7812.

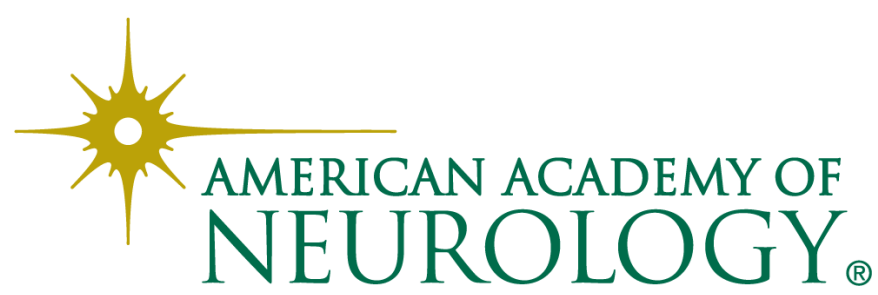




\section{Updated Information \& Services}

References

Subspecialty Collections

Permissions \& Licensing

\section{Reprints}

including high resolution figures, can be found at: http://nn.neurology.org/content/9/2/e1135.full.html

This article cites 39 articles, 2 of which you can access for free at: http://nn.neurology.org/content/9/2/e1135.full.html\#\#ref-list-1

This article, along with others on similar topics, appears in the following collection(s):

\section{Class I}

http://nn.neurology.org//cgi/collection/class_1

\section{MRI}

http://nn.neurology.org//cgi/collection/mri

Optic nerve

http://nn.neurology.org//cgi/collection/optic_nerve

Optic neuritis; see Neuro-ophthalmology/Optic Nerve

http://nn.neurology.org//cgi/collection/optic_neuritis

\section{Retina}

http://nn.neurology.org//cgi/collection/retina

Information about reproducing this article in parts (figures,tables) or in its entirety can be found online at:

http://nn.neurology.org/misc/about.xhtml\#permissions

Information about ordering reprints can be found online: http://nn.neurology.org/misc/addir.xhtml\#reprintsus

Neurol Neuroimmunol Neuroinflamm is an official journal of the American Academy of Neurology.

Published since April 2014, it is an open-access, online-only, continuous publication journal. Copyright

Copyright $\odot 2022$ The Author(s). Published by Wolters Kluwer Health, Inc. on behalf of the American Academy of Neurology.. All rights reserved. Online ISSN: 2332-7812.

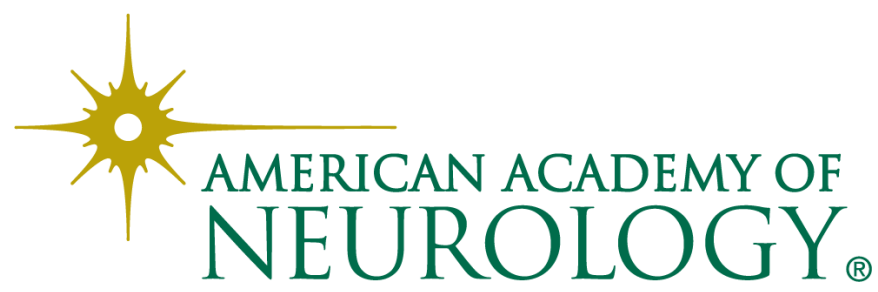

